\title{
Evaluation of standard of care intravitreal aflibercept treatment of diabetic macular oedema treatment-naive patients in the UK: DRAKO study 12-month outcomes
}

\author{
Sobha Sivaprasad (iD ${ }^{1 凶}$, Faruque Ghanchi (iD $^{2}$, Simon P. Kelly ${ }^{3}$, Ajay Kotagiri ${ }^{4}$, James Talks (DD $^{5}$, Peter Scanlon (iD $^{6}$, Hellen McGoey ${ }^{7}$, \\ Andrew Nolan $\mathbb{D}^{7}$, Moneeb Saddiq ${ }^{8}$, Jackie Napier ${ }^{7}$ on behalf of the DRAKO study group
}

(c) The Author(s) 2021

\begin{abstract}
OBJECTIVES: DRAKO (NCT02850263) is a 24-month, prospective, non-interventional, multi-centre cohort study which enroled patients diagnosed with centre-involving diabetic macular oedema (DMO). The study aims to evaluate standard of care with intravitreal aflibercept (IVT-AFL) treatment in the UK. This analysis describes the anti-vascular endothelial growth factor (anti-VEGF) treatment-naive patient cohort after 12-month follow-up.

METHODS: Study eyes were treated with IVT-AFL as per local standard of care. The mean change in best-corrected visual acuity (BCVA) and central subfield thickness (CST) from baseline at 12 months were measured and stratified by baseline factors. The number of injections and safety data were also evaluated.

RESULTS: A total of 507 patients were enroled from 35 centres. Mean (SD) baseline BCVA was 71.4 (12.0) letters and CST was 448.7 (88.7) $\mu \mathrm{m}$, with $63.1 \%$ of patients presenting with baseline BCVA $\geq 70$ letters (mean 78.1). Mean (SD) change in BCVA of 2.5 (12.2) letters and CST of -119.1 (116.4) $\mu \mathrm{m}$ was observed at month 12. A 7.3 letter gain was observed in patients with baseline BCVA $<70$ letters. Mean number (SD) of injections in year one was 6.4 (2.1). No significant adverse events were recorded.

CONCLUSION: Year one results indicated that IVT-AFL was an effective treatment for DMO in standard of care UK clinical practice, maintaining or improving visual acuity in treatment-naive patients with good baseline visual acuity, despite some patients being undertreated versus the summary of product characteristics. These results also demonstrated the clinical importance and meaningful impact of diabetic retinopathy screening in the UK.
\end{abstract}

Eye (2022) 36:64-71; https://doi.org/10.1038/s41433-021-01624-9

\section{INTRODUCTION}

Diabetes, including its associated complications, is an escalating healthcare problem, with treatment estimated to cost around $10 \%$ of the UK's entire National Health Service (NHS) budget [1]. The global prevalence of diabetes is projected to increase from the current $9.3 \%$ to $10.2 \%$ in 2030 , affecting 578.4 million adults between the ages of 20-79 years, further increasing the burden on health systems [2]. Diabetic macular oedema (DMO), a microvascular complication of diabetes that can occur at any stage of retinopathy, is the most common cause of visual acuity (VA) loss in patients with diabetes and the most frequent cause of blindness in young and mid-aged adults in the developed world. One in four diabetic patients can expect to develop DMO in their lifetime [3-6].

In recent years, the use of anti-vascular endothelial growth factor (anti-VEGF) treatments for DMO has seen outcomes for many patients improve. Intravitreal injections of anti-VEGF therapies have shown superiority over laser photocoagulation of the macula in several clinical trials [7-10]. Subsequently, intravitreal VEGF inhibitors have become the first-line therapy of choice for management of vision loss from DMO.

Intravitreal aflibercept (IVT-AFL, Eylea) is an anti-VEGF treatment with an innovative fusion protein design. Current IVT-AFL standard of care treatment for DMO patients is a $2 \mathrm{mg}$ dose of five initial monthly injections followed by a proactive bi-monthly treatment regimen in year one, with no requirement for monitoring between injections. In year two, the injection interval may be extended dependent on functional and anatomical outcomes, consequently reducing burden on patients and their care providers (IVT-AFL summary of product characteristics [SmPC] recommended posology for DMO treatment) [11].

Results from the two pivotal IVT-AFL Phase 3 trials in DMO, VIVID ${ }^{\mathrm{DMO}}$ and VISTA ${ }^{\mathrm{DMO}}$, demonstrated significant superiority for the IVT-AFL treatment groups over laser in all endpoints at week 52 through to week 148 , with similar efficacy in both treatment regimens $[10,12,13]$.

The UK did not participate in these pivotal trials, and DRAKO represents the first UK-based prospective, non-interventional

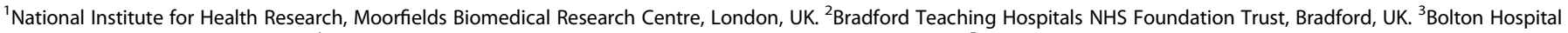

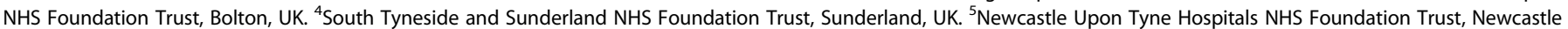

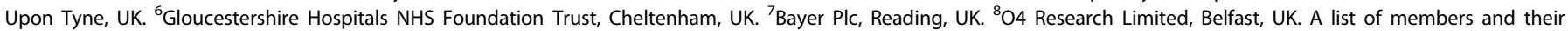
affiliations appears in the Supplementary Information. ${ }^{凶}$ email: senswathi@aol.com 
Table 1. Patient inclusion and exclusion criteria based on cohort for the DRAKO non-interventional study.

\author{
Inclusion criteria \\ Universal study inclusion criteria \\ Aged 18 years or older \\ Type 1 or 2 diabetes mellitus diagnosis
}

DMO diagnosis with central involvement

Decision to initiate IVT-AFL treatment made as per routine practice and before study inclusion

Written informed consent

\section{Exclusion criteria}

Contraindications as listed in the SmPC for IVT-AFL

Pre-planned cataract surgery during the observational period

Currently or previously treated with systemic anti-VEGF

Previously treated with intravitreal fluocinolone acetonide steroid

Participating in an investigational programme with interventions outside of routine clinical practice

Women who are currently pregnant or lactating and/or planning pregnancy within the next 2 years

Known hypersensitivity to any excipients

Active or suspected ocular/periocular infection or periocular inflammation

DMO diabetic macular oedema, IVT-AFL intravitreal aflibercept, anti-VEGF anti-vascular endothelial growth factor, SmPC summary of product characteristics.
Anti-VEGF treatment-naive cohort specific criteria

Not previously received anti-VEGF treatment

Not received pan-retinal photocoagulation treatment in the past 8 weeks

Not received macular laser photocoagulation treatment in the past 4 months

Not previously received intravitreal dexamethasone steroid or fluocinolone acetonide treatment

Prior Anti-VEGF treatment other than IVT-AFL cohort specific criteria

Previously treated with an intravitreal anti-VEGF other than aflibercept for DMO

Not received intravitreal anti-VEGF treatment in the past 28 days

Not received intravitreal dexamethasone steroid treatment in the past 6 months study to assess the effectiveness of standard of care IVT-AFL treatment in DMO patients. Such observational studies are valuable as they report and evaluate outcomes based on locally defined treatment practices, outside the rigorous clinical trial setting, enabling outcome characterisation within a more representative population and treatment environment.

The primary objectives of this study are to assess the mean change from baseline in best-corrected visual acuity (BCVA) and central subfield thickness (CST). In addition, by evaluating followup procedures and assessing treatment patterns in eyes affected by DMO treated with IVT-AFL in routine clinical practice, the study aims to inform best practice guidance in the UK upon completion of the follow-up period. Here we describe the month 12 outcomes of the anti-VEGF treatment-naive patient cohort.

\section{MATERIALS AND METHODS \\ Study design}

DRAKO (NCT02850263) is a prospective, non-interventional, multi-centre, non-comparative cohort study conducted in $35 \mathrm{NHS}$ hospitals throughout the UK (Supplementary Table 1). Patients were enroled from July 2016 through to April 2018 and followed up for 24 months. To reduce selection bias, patients were enroled in a consecutive manner. The study was approved by the North West Liverpool East Research Ethics Committee (16/NW/0238) and was conducted in accordance with the Declaration of Helsinki. All participants provided written informed consent. All treatment decisions, including the decision to treat with IVT-AFL, were made as per local standard of care, independently of study participation. The study included two patient cohorts; Cohort 1 comprised anti-VEGF treatment-naive patients and Cohort 2 comprised patients who previously received anti-VEGF treatment other than IVTAFL for DMO. Study size was calculated based on an assumed 10\% dropout rate at 24 months, allowing an estimate of change from baseline in BCVA letters within $95 \%$ confidence intervals $(\mathrm{Cl})$ of \pm 1.2 letters and CST of $\pm 13.9 \mu \mathrm{m}$. This was based on a standard deviation (SD) of 13 letters for the mean change in BCVA and $150 \mu \mathrm{m}$ for CST, conservative estimates based on SD observed in other recent studies involving aflibercept in
DMO [10, 14]. Sample sizes of 450 and 225 were calculated for Cohorts 1 and 2 respectively.

\section{Study population and treatment}

Adult patients with a confirmed diagnosis of type 1 or type 2 diabetes and DMO with central involvement, defined as the centre subfield area on optical coherence tomography, were enroled if they met the eligibility criteria as defined in Table 1. Inclusion of patients based on a CST of $\geq 400 \mu \mathrm{m}$ in the study eye was removed as an eligibility criterion via protocol amendment (21 February 2017) as this threshold is not applied in Scotland and removal enabled a more accurate representation of the UK-treated population. One of the two participating Scottish sites (University Hospital Hairmyres) was active prior to approval of the protocol amendment. The study eye was defined as the eye for which the IVT-AFL treatment was initiated or, if both eyes were affected, the eye with worse baseline VA. Data were collected for the fellow eye if DMO diagnosis was confirmed. No eligibility restriction was mandated for patient baseline BCVA letter score.

At study initiation, details of the local standard of care IVT-AFL treatment protocol was recorded for each centre (Supplementary Table 2). Data collection, including type of visit; diabetic management (diabetic retinopathy measured using the English National Screening Committee or Scottish Diabetic Retinopathy Grading Scheme classifications, and haemoglobin $\mathrm{A}_{1 \mathrm{c}}\left(\mathrm{HbA}_{1 \mathrm{c}}\right)$ assessment), anatomical and functional assessments; treatment provided from the baseline visit; and all subsequent routine outpatient visits were recorded. The month 12 visit was nominated by the site (for data collection purposes) and defined as 12 months \pm 1 month from the patient's baseline visit.

Patients who dropped out of the study for any reason were not replaced.

\section{Outcome measures}

Primary outcomes were the mean change from baseline in Early Treatment Diabetic Retinopathy Study (ETDRS) letters measured by BCVA with refraction and the mean change in CST as determined by spectral domain optical coherence tomography (as per local practice) at month 12. Secondary objectives included mean change in BCVA and CST stratified by pre-defined baseline factors; percentage gain and loss of at least 5, 10 or 15 letters; and adherence to IVT-AFL DMO SmPC in year one. Due to the observational design of the study, target changes in these parameters were not defined. 


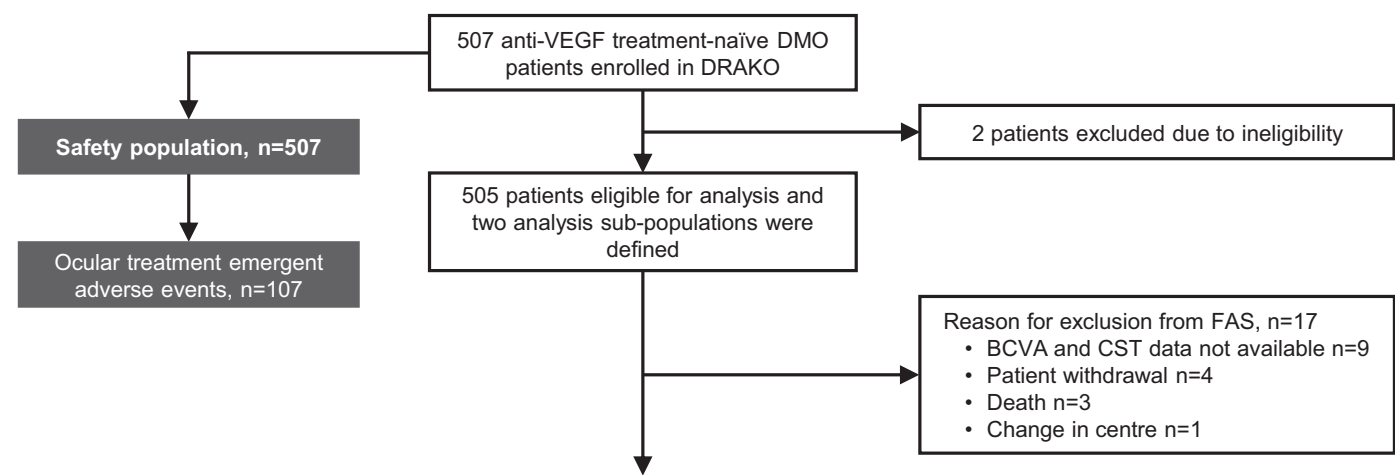

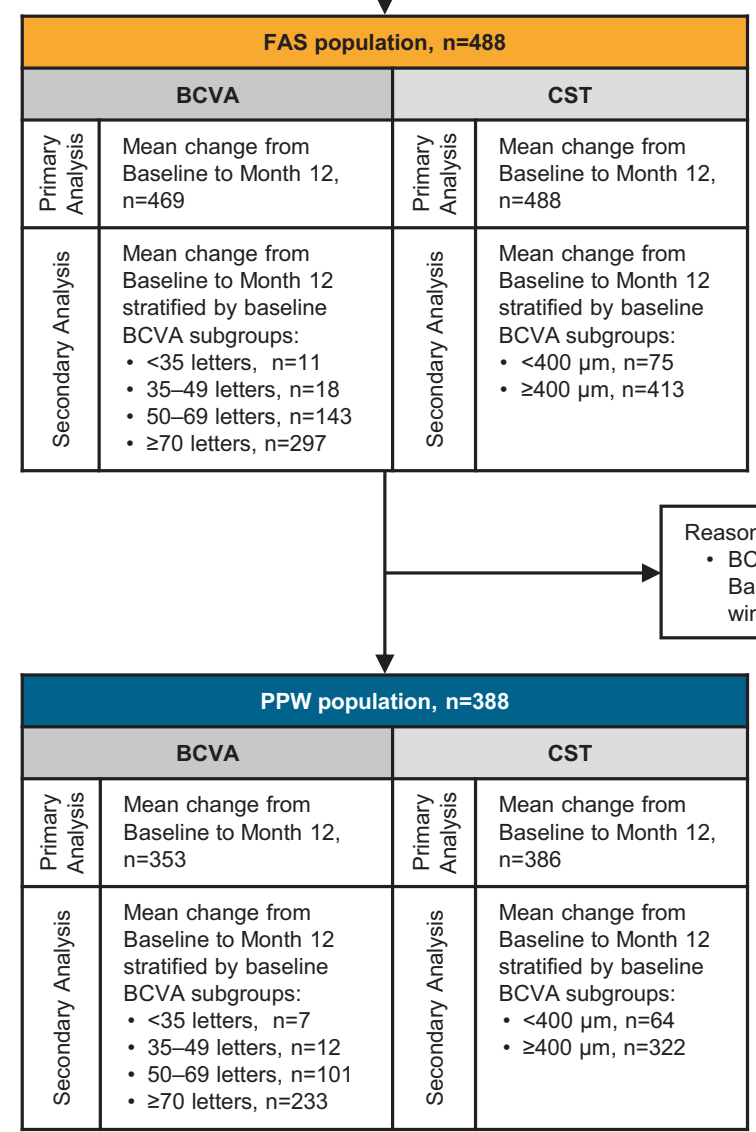

Fig. 1 Patient disposition during the study and analysis summary. DMO diabetic macular oedema, anti-VEGF anti-vascular endothelial growth factor, IVT-AFL intravitreal aflibercept, PPW per protocol window population, FAS full analysis set, BCVA best-corrected visual acuity, CST central subfield thickness, $\mathrm{n}$ number of patients per group.

\section{Statistical analysis}

Interim analysis was conducted upon completion of the 12-month followup period for the treatment-naive patient cohort and are reported here.

Descriptive statistics were used to summarise the quantitative variables. Categorical variables were summarised using frequency distributions and percentages and data stratified by pre-defined baseline covariate subgroups; age (18-35, 36-50, 51-65, >65 years), BCVA $(<35,35-49$, $50-69, \geq 70$ letters) and CST $(<400, \geq 400 \mu \mathrm{m})$.

All patients with a baseline IVT-AFL injection and at least one postbaseline assessment of BCVA or CST were included in the analysis. Two sub-populations were analysed, and $95 \% \mathrm{Cl}$ were calculated. The more stringent sub-population, defined as the per protocol window population (PPW), included patients with available BCVA or CST data at baseline and the nominated month 12 visit (12 months \pm 1 month). The less stringent sub-population was defined as the full analysis set (FAS) and included patients with BCVA or CST available at baseline and at least one follow-up visit; missing values were imputed based on the last observation carried forward method, the visit immediately preceding the month 12 visit was used for analysis. Adherence to SmPC was measured by defining acceptable 'windows' between injections. For the first five injections, it was 25-38 days; after the fifth injection, it was 46-66 days.

Safety was assessed on the safety set, which included all patients who provided written informed consent. Adverse events were listed using the Medical Dictionary for Regulatory Activities coding system.

Analysis was performed using SAS ${ }^{\star}$ software, version 9.4 (SAS Institute Inc., Cary, NC, USA).

\section{RESULTS}

\section{Patient disposition and baseline characteristics}

DRAKO enroled 507 anti-VEGF treatment-naive patients. A 2.0\% dropout rate was observed in year one, the reasons for drop-out were as follows; patient withdrawal (4), death (3), patient ineligibility (2) and change in centre (1). Further patients were excluded from the analysis due to unavailability of baseline and/or 12-month data. Patients were 
Table 2. Patient demographics and baseline characteristics per subpopulation.

\begin{tabular}{|c|c|c|}
\hline & PPW & FAS \\
\hline \multicolumn{3}{|l|}{ Age, years, mean (SD) } \\
\hline All & $62.9(11.6)$ & $62.4(11.6)$ \\
\hline $18-35$ & $30(4.3)$ & $29.4(4.4)$ \\
\hline $36-50$ & $45.2(3.5)$ & $45.6(3.4)$ \\
\hline $51-65$ & $58.9(4.3)$ & $58.7(4.2)$ \\
\hline$>65$ & $73.4(5.9)$ & $73.3(5.8)$ \\
\hline \multicolumn{3}{|l|}{ Sex, $n(\%)$} \\
\hline Male & $245(63.1)$ & $303(62.1)$ \\
\hline Female & $143(36.9)$ & 185 (37.9) \\
\hline \multicolumn{3}{|l|}{ Race or ethnicity, $n$ (\%) } \\
\hline White & $298(76.8)$ & $374(76.6)$ \\
\hline Asian & $38(9.8)$ & $50(10.2)$ \\
\hline Black & $29(7.5)$ & $38(7.8)$ \\
\hline Mixed & $4(1.0)$ & $4(0.8)$ \\
\hline Hispanic & $2(0.5)$ & $3(0.6)$ \\
\hline Unknown & $14(3.6)$ & $15(3.1)$ \\
\hline Other & $3(0.8)$ & $4(0.8)$ \\
\hline \multicolumn{3}{|l|}{ Diabetes type, $n(\%)$} \\
\hline 1 & $55(14.2)$ & $67(13.7)$ \\
\hline 2 & $333(85.8)$ & $421(86.3)$ \\
\hline \multicolumn{3}{|l|}{ Diabetic retinopathy, $n$ (\%) } \\
\hline English Scale & $265(85.8)$ & $340(87.4)$ \\
\hline Ro & $4(1.5)$ & $5(1.5)$ \\
\hline $\mathrm{R} 1$ & $111(41.9)$ & $142(41.8)$ \\
\hline $\mathrm{R} 2$ & $104(39.2)$ & $133(39.1)$ \\
\hline R3 & $46(17.4)$ & $60(17.6)$ \\
\hline Scottish Scale & $44(14.2)$ & $49(12.6)$ \\
\hline Ro & 0 & 0 \\
\hline $\mathrm{R} 1$ & $21(47.7)$ & $25(51.0)$ \\
\hline $\mathrm{R} 2$ & $21(47.7)$ & $21(42.9)$ \\
\hline R3 & $2(4.5)$ & $3(6.1)$ \\
\hline R4 & 0 & 0 \\
\hline \multicolumn{3}{|l|}{$\mathrm{HbA} 1 \mathrm{c}(\mathrm{mmol} / \mathrm{mol}), n$ mean (SD) } \\
\hline$n$ & 194 & 239 \\
\hline Mean (SD) & $66.1(20.5)$ & $66.2(20.3)$ \\
\hline \multicolumn{3}{|l|}{ Fellow eye involvement, $n$ (\%) } \\
\hline DMO confirmed in fellow eye & $209(53.9)$ & $265(54.3)$ \\
\hline $\begin{array}{l}\text { Diabetes diagnosis to baseline, mean } \\
\text { (SD) years }\end{array}$ & $16.0(10.5)$ & $15.8(10.3)$ \\
\hline $\begin{array}{l}\text { DMO diagnosis to baseline, years, } \\
\text { mean (SD) }\end{array}$ & $1.3(2.4)$ & $1.3(2.4)$ \\
\hline
\end{tabular}

$S D$ standard deviation, $n$ number of patients, PPW per protocol window population, FAS full analysis set, DMO diabetic macular oedema.

divided into PPW $(n=388)$ and FAS $(n=488)$ sub-populations for analysis based on pre-defined stringency criteria (Fig. 1). The PPW and FAS sub-populations demonstrated comparable trends throughout the analysis and results for the PPW sub-population are reported unless stated otherwise.

The mean age at baseline was 62.9 years, and $63.1 \%$ of patients were male (Table 2). Most patients were white (76.8\%), and $85.8 \%$ had been diagnosed with type 2 diabetes. Over half of patients had fellow eye involvement. Patients demonstrated a suboptimal level of glycaemic control with mean (SD) $\mathrm{HbA}_{1 \mathrm{c}}$ of 66.1 (20.5) $\mathrm{mmol} / \mathrm{mol}$. Background to pre-proliferative retinopathy was recorded for the majority of patients regardless of classification applied. On average, patients had been diagnosed with DMO 1.3 (SD 2.4) years before their baseline visit. Of the primary endpoint assessments, BCVA was recorded for $91.0 \%$ of patients and CST for 99.5\% of patients. The mean (SD) baseline BCVA and CST were 71.4 (12.0) letters and 448.7 (88.7) $\mu \mathrm{m}$, respectively (Table 3).

\section{Treatment of DMO}

Local standard of care IVT-AFL treatment was administered across all sites, with 11 different treatment posologies recorded at study initiation (Supplementary Table 2). All patients received at least one IVT-AFL injection in the study eye. The mean number (SD) of injections was 6.4 (2.1) and 6.3 (2.2) for the PPW and FAS subpopulations, respectively, compared with eight to nine injections if treatment was conducted as per the SmPC. At site initiation, 27 $(77.1 \%)$ and $21(60.0 \%)$ of the 35 participating sites confirmed their intention to adhere to DMO SmPC for the five initial monthly injections and for 12 months respectively as per their local standard of care protocol. This equated to 334 patients $(86.1 \%)$ from the 27 sites intending to administer five initial injections and $211(54.4 \%)$ from the 21 sites intending to adhere to SmPC throughout year one. However, in practice, 117 (30.2\%) of patients received five initial IVT-AFL injections and 13 (3.4\%) patients adhered to SmPC in year one.

\section{Effect of treatment on functional and anatomical outcomes}

The mean $(95 \% \mathrm{Cl})$ improvement in BCVA at month 12 was 2.5 letters $( \pm 1.3$ letters) and 1.9 letters ( \pm 1.0 letters) for the PPW and FAS sub-populations, respectively (Table 3 ). The relative increase in BCVA letters varied based on initial VA. Patients with baseline BCVA of less than 35 letters benefitted from a mean increase of 23.6 letters $(n=7)$, whereas the mean BCVA for patients presenting with BCVA of 70 letters or more remained stable (Fig. 2A and Supplementary Table 3). Similarly, patients aged 18-35 years experienced a larger increase in mean BCVA letters than those aged 65 years and older (4.3 and 1.2 letters, respectively; Supplementary Table 3). A letter gain of five or more was observed in $40.2 \%$ of patients, whereas an equivalent letter loss was noted in $16.5 \%$ patients (Fig. 2B). When evaluated by adherence to SmPC, patients receiving five initial monthly injections obtained a mean (SD) letter gain of 4.2 (10.9). The small proportion of patients for whom treatment continued as per SmPC in year one experienced a 1.1 (7.4) letter gain (Supplementary Table 4).

At month 12 , CST was reduced, with mean $(95 \% \mathrm{Cl}$ ) change from baseline determined as $-119.1 \mu \mathrm{m}( \pm 11.6 \mu \mathrm{m})$ and $-117.7 \mu \mathrm{m}$ $( \pm 10.1 \mu \mathrm{m})$ for the PPW and FAS sub-populations, respectively (Table 3). Baseline CST was $<400 \mu \mathrm{m}$ for $16.5 \%$ of patients. Mean (SD) CST change was observed as $-42.5(76.3) \mu \mathrm{m}$ and -134.3 (117.0) $\mu \mathrm{m}$ for patients with initial CST measure of $<400 \mu \mathrm{m}$ and $\geq 400 \mu \mathrm{m}$, respectively (Fig. $2 \mathrm{C}$ and Supplementary Table 5). A larger improvement in BCVA was observed at month 12 for patients presenting with a baseline CST of $\geq 400 \mu \mathrm{m}$ (mean [SD] BCVA letter change was 1.4 [9.2] and 2.7 [12.6] for patients with CST of $<400 \mu \mathrm{m}$ and $\geq 400 \mu \mathrm{m}$, respectively; see Supplementary Table 3).

\section{Adverse events}

The safety set comprised data from the full treatment-naive patient cohort $(n=507)$. During the first 12 months of treatment, a total of 3129 injections were administered to the study eye. Nonocular treatment emergent adverse events (TEAE) were reported for $33.7 \%(n=171)$ of patients and ocular TEAEs for $13.2 \%(n=67)$ of patients. A total of 107 ocular TEAEs were captured. Reported TEAEs (percentage of patients) included vitreous haemorrhage $(3.0 \%)$, cataract $(1.2 \%)$, retinal haemorrhage $(1.2 \%)$, retinal 
Table 3. Mean change from baseline in functional and anatomical endpoints at month 12 .

\begin{tabular}{|c|c|c|c|c|c|c|}
\hline \multirow[t]{2}{*}{ Assessment } & \multicolumn{3}{|c|}{ Per protocol window $(n=\mathbf{3 8 8})$} & \multicolumn{3}{|c|}{ Full analysis set $(n=488)$} \\
\hline & Mean (SD) & $95 \% \mathrm{Cl}$ & Patient number & Mean (SD) & $95 \% \mathrm{Cl}$ & Patient number \\
\hline \multicolumn{7}{|l|}{ BCVA } \\
\hline Month 12 & $73.6(13.9)$ & NA & 362 & $72.5(14.3)$ & NA & 481 \\
\hline Change from baseline & $2.5(12.2)$ & $1.3,3.8$ & 353 & $1.9(11.3)$ & $0.9,2.9$ & 469 \\
\hline \multicolumn{7}{|l|}{ CST } \\
\hline Month 12 & $329.8(92.3)$ & NA & 386 & $334.4(94.1)$ & NA & 488 \\
\hline Change from baseline & $-119.1(116.4)$ & $-130.7,-107.4$ & 386 & $-117.7(113.7)$ & $-127.8,-107.6$ & 488 \\
\hline
\end{tabular}

Baseline and Month 12 mean (SD) for the primary endpoints with patient number defined per analysis sub-population. Mean change in BCVA and CST from Baseline were reported, and $95 \% \mathrm{Cl}$ were calculated.

SD standard deviation, BCVA best-corrected visual acuity, CST central subfield thickness, Cl confidence interval, NA not applicable.

detachment $(0.2 \%)$ and vitreous detachment (0.2\%). Adverse events reported as associated with treatment were primarily related to the procedure rather than aflibercept. The safety profile was in line with other published studies, and no new safety concerns were identified. Further details on reported TEAEs are outlined in Supplementary Table 6.

\section{DISCUSSION}

DRAKO is the first prospective, multi-centre study evaluating standard of care IVT-AFL treatment for DMO in the UK. The study enroled 507 treatment-naive patients across 35 contributing centres and it was noted that the majority of patients with DMO are diagnosed and treated at a high level of VA, demonstrating the effectiveness of the diabetic retinopathy screening programme in the UK.

The mean baseline BCVA for DRAKO (71.4 letters) was substantially higher than measures reported in pivotal clinical trials (VIVID ${ }^{\mathrm{DMO}}$ and VISTA ${ }^{\mathrm{DMO}}$ aflibercept cohorts reported mean baseline BCVA of 59.8 letters and 59.1 letters, respectively) and in other prospective observational DMO studies (APOLLON and POLARIS) $[10,15,16]$. Of note, two-thirds of DRAKO patients had baseline BCVA of 70 letters or more (mean 78.1). APOLLON, a French IVT-AFL treatment-based study enroled 77 patients between September 2016 and July 2019, reported mean baseline VA 62.7 letters in the treatment-naive cohort [15]. However, eligibility was limited to patients with baseline VA $\leq 20 / 40 \quad(\leq 70$ ETDRS letters). POLARIS, a European-based, ranibizumab study that recruited 125 patients within the UK cohort from September 2012 to January 2015 reported a mean baseline VA of 60.3 letters [16]. When comparing baseline VA in these UK cohorts, a clinically relevant difference of 11 letters in baseline VA was observed between DRAKO and POLARIS. Although these letter changes are not directly comparable given that DRAKO captured BCVA with refraction and POLARIS documented non-refracted VA, these measures are nonetheless highly correlated. This may be a further indication of a shift towards earlier screening and treatment of DMO within the NHS in more recent years, particularly as DRAKO observed a mean time from diagnosis of DMO to baseline treatment of 1.3 years, suggesting that patients are often being diagnosed with DMO in advance of the condition being considered clinically significant. Indeed, these results suggest that the National Institute for Health and Care Excellence (NICE) Technical Appraisal Guidance on the threshold for treatment initiation (central retinal thickness of at least $400 \mu \mathrm{m}$ ) may be encouraging early intervention to maintain or improve patient VA in areas of the UK where these guidelines apply (England, Wales and Northern Ireland).
DRAKO patients experienced a mean gain of 2.5 letters and a mean reduction in CST of $119.1 \mu \mathrm{m}$ at month 12. Trends in letter gains and losses demonstrated that $40.2 \%$ and $20.1 \%$ patients observed at least a 5 - or 10-letter gain respectively within the first year. Whereas, $16.5 \%$ and $9.8 \%$ patients observed at least a 5 - or 10-letter loss respectively within the same period. A 5-letter gain in the context of the high observed baseline BCVA scores may be clinically relevant in most patients. In addition, stratification by baseline BCVA demonstrated that better gains were achieved in those with worse baseline BCVA. Of note, for patients with a baseline BCVA $\leq 69$ letters (mean 58.7 letters), the mean gain at month 12 was 7.3 letters.

Although these trends reflect those seen in both IVT-AFL treatment randomised clinical trials (VIVID ${ }^{\mathrm{DMO}}$ and VISTA ${ }^{\mathrm{DMO}}$ ) and the APOLLON observational study, the overall 12-month BCVA letter gains observed in these studies are higher than in DRAKO (gain of 10.7, 10.7 and 7.8 letters, respectively) [10, 15]. This variation is likely due to the protocol-driven higher injection numbers, particularly in the pivotal trials (patients received five initial monthly doses followed by IVT-AFL treatment every 8 weeks) and the increased opportunity for larger BCVA gains from a lower baseline as a result of implementing a maximum BCVA inclusion criteria which was not mandated for DRAKO.

Previous reports have highlighted the positive association of injection frequency and functional outcomes for patients, with an emphasis on the initial injections [17]. The DRAKO study authors also advocate the administration of full initial dosing, with the subgroup of patients receiving five initial monthly injections obtaining a mean letter gain of 4.2, although this benefit was not extended to year one SmPC compliance, potentially due to the low patient numbers or high baseline BCVA. The reasons for the lower than intended number of injections administered were not collected and changes in standard of care treatment protocols at participating sites were not re-assessed after site initiation. Consequently, it was not possible to determine whether this was due primarily to capacity constraints within the NHS or other factors, such as clinical prioritisation, patient influences or perhaps a more relaxed treatment approach in response to favourable baseline metrics. Of note, APOLLON results showed a higher rate of patients receiving five initial doses of IVT-AFL (51.9\%) than DRAKO, which may also have contributed to the higher letter gain reported.

Overall, DRAKO patients with a baseline BCVA of $\geq 70$ letters maintained their vision. Those with baseline BCVA of 70-78 letters $(n=139)$ gained 1.3 letters, and those with baseline BCVA $>78$ $(n=94)$ experienced a loss of 1.8 letters. This distinction may be important in efforts to preserve 'driving vision', which is categorised in the UK as a BCVA of 70 letters or more in the 

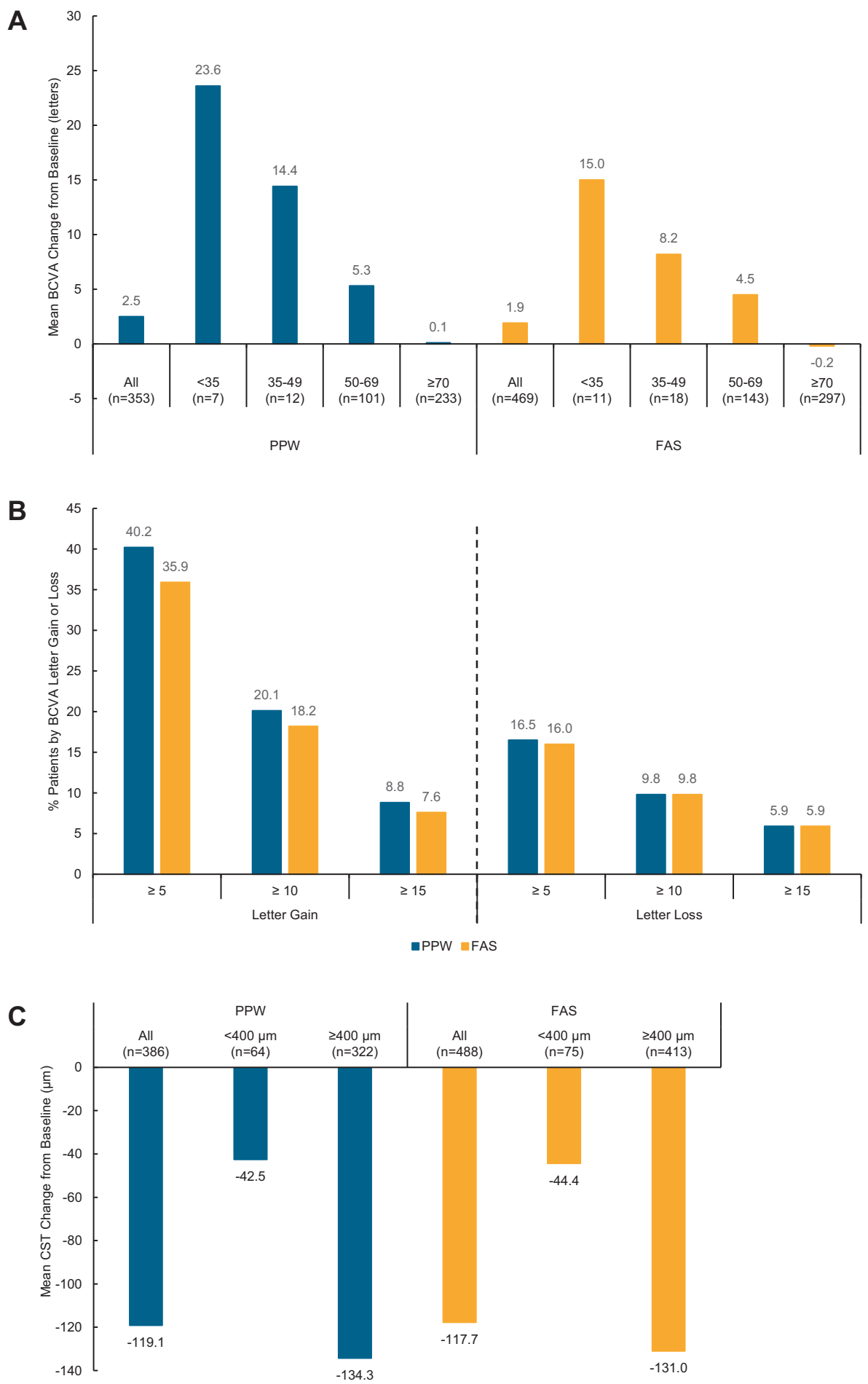

Fig. 2 Primary outcome results stratified by baseline measure and BCVA letter gain or loss proportion. A BCVA stratification, B BCVA letter gain and loss proportion and C CST stratification, by sub-population. BCVA best-corrected visual acuity, CST central subfield thickness, PPW per protocol window population, FAS full analysis set.

patient's better eye. The results for patients with BCVA 78 letters or more are comparable to those of the Diabetic Retinopathy Clinical Research Retina Network Protocol V study. Whilst no control group was included in the DRAKO study design, the reduced benefits observed in this subgroup support the conclusion of Protocol $V$ that a reasonable strategy for patients with high VA may be observation without treatment unless VA worsens, although there were few patients with $>400 \mu \mathrm{m}$ CST in Protocol V
(9\% patients in the aflibercept group) [18]. Analysis of the DRAKO year 2 results may provide further clarity on this clinically important point.

These DRAKO observations are supported by several retrospective registry studies, which demonstrated the real-world effectiveness of IVT-AFL for DMO treatment [19-21]. Notably, fewer injections were recorded compared to clinical trials, and functional gains were not as pronounced and were related to 
baseline measures. In the UK-based, single-centre retrospective cohort study conducted by Lukic et al. [21]., 99 eyes were evaluated and a mean of 6.9 injections were administered. The baseline VA (59.7 ETDRS letters) in this study was much lower than DRAKO, so although the overall letter gain (9.9 letters) was higher than that observed in DRAKO, in the $34 \%$ of eyes with a baseline $V A \geq 69$, the mean gain was 2.6 letters.

A similar 'ceiling' effect was observed in the CST subgroup analysis. Baseline CST was stratified above or below $400 \mu \mathrm{m}$ to align with the NICE Technology Appraisal Guidance. The reduction in CST for the baseline $\geq 400 \mu \mathrm{m}$ subgroup was much greater than for the $<400 \mu \mathrm{m}$ subgroup, but the mean CST at month 12 was similar for both subgroups. The results suggest minimal difference in terms of BCVA outcomes between the two subgroups, with a marginally lower letter gain attained in the $<400 \mu \mathrm{m}$ subgroup, although patient numbers were limited $(n=52)$.

The study has some limitations often inherent in noninterventional studies such as, inconsistent treatment administration and non-defined functional eligibility metrics. However, the prospective study design and wide range of sites contributing enables treatment effects to be monitored across a diverse population that aims to be representative of the UK as a whole. In addition, the large number of anti-VEGF treatment-naive patients enroled, and the high proportion of primary endpoint data collected, strengthen the observations made during the study.

In conclusion, year one results indicate that IVT-AFL is an effective treatment for DMO in real-world UK clinical practice, maintaining or improving VA. Vision gains observed in DRAKO were lower than in the pivotal clinical trials and several observational studies [10, 14-16], perhaps reflecting better baseline vision in UK clinical practice and/or a failure to adhere to the SmPC for all patients. In addition, DRAKO demonstrated the effectiveness of diabetic retinopathy screening in the UK, with almost two-thirds of patients presenting with good baseline vision.

The DRAKO data set contains a rich source of real-world ocular-, treatment- and resource-related data that will mature further upon completion of the study. It is anticipated that this will provide a benchmark for better understanding of DMO IVT-AFL outcomes and for shaping how DMO patient care pathways are defined in the future.

\section{SUMMARY}

What was known before

- The effectiveness of intravitreal aflibercept (IVT-AFL) for treatment of diabetic macular oedema (DMO) patients has been demonstrated in several pivotal clinical trials (VIVID and VISTA) and non-UK focused observational studies (APOLLON), although such investigations primarily focused on patients with baseline visual acuity of $<73$ letters.

- Retrospective registry-based studies of anti-vascular endothelial growth factor (anti-VEGF) treatments have reported lower injection frequency and functional gains than randomised clinical trials.

- The scope for improvement of functional and anatomical parameters in response to anti-VEGF treatment is closely associated with baseline values.

What this study adds

- DRAKO is the first prospective non-interventional study to evaluate IVT-AFL treatment of DMO patients across the UK, demonstrating the effectiveness of this treatment to maintain or improve patient outcomes across a diverse range of local standard of care protocols, despite often observing undertreatment compared with locally defined treatment plans.

- DRAKO indicates the effectiveness of the diabetic retinopathy screening programme in the UK where patients with DMO are being identified and treated at a high level of VA, thereby preserving patient vision.

\section{REFERENCES}

1. Written by Editor. Diabetes U. K. Cost of diabetes. 2019 https://www.diabetes.co. uk/cost-of-diabetes.html. Accessed 19 Nov 2020.

2. International Diabetes Federation Diabetes Atlas ninth edition. 2019 https:// diabetesatlas.org/upload/resources/2019/IDF_Atlas_9th_Edition_2019.pdf. Accessed 26 Nov 2020.

3. Fong DS, Aiello LP, Ferris FL 3rd, Klein R. Diabetic retinopathy. Diabetes Care. 2004;27:2540-53.

4. Musat $O$, Cernat $C$, Labib M, Gheorghe A, Toma O, Zamfir M, et al. Diabetic macular edema. Rom J Ophthalmol. 2015;59:133-6.

5. Moss SE, Klein R, Klein BE. The 14-year incidence of visual loss in a diabetic population. Ophthalmology. 1998;105:998-1003.

6. Stefánsson E, Bek T, Porta M, Larsen N, Kristinsson JK, Agardh E. Screening and prevention of diabetic blindness. Acta Ophthalmol Scand. 2000;78:374-85.

7. Nguyen QD, Brown DM, Marcus DM, Boyer DS, Patel S, Feiner L, et al. Ranibizumab for diabetic macular edema: results from 2 phase III randomized trials: RISE and RIDE. Ophthalmology. 2012;119:789-801.

8. Mitchell $P$, Bandello F, Schmidt-Erfurth U, Lang GE, Massin P, Schlingemann RO, et al. The RESTORE study: ranibizumab monotherapy or combined with laser versus laser monotherapy for diabetic macular edema. Ophthalmology. 2011;118:615-25.

9. Do DV, Nguyen QD, Boyer D, Schmidt-Erfurth U, Brown DM, Vitti R, et al. One-year outcomes of the da Vinci Study of VEGF Trap-Eye in eyes with diabetic macular edema. Ophthalmology. 2012;119:1658-65.

10. Korobelnik JF, Do DV, Schmidt-Erfurth U, Boyer DS, Holz FG, Heier JS, et al. Intravitreal aflibercept for diabetic macular edema. Ophthalmology. 2014;121:2247-54.

11. Eylea $40 \mathrm{mg} / \mathrm{ml}$ solution of injection in a vial summary of product characteristics. 2012. https://www.medicines.org.uk/emc/product/2879/smpc\#gref. Accessed 19 Nov 2020.

12. Brown DM, Schmidt-Erfurth U, Do DV, Holz FG, Boyer DS, Midena E, et al. Intravitreal aflibercept for diabetic macular edema: 100-week results from the VISTA and VIVID studies. Ophthalmology. 2015;122:2044-52.

13. Heier JS, Korobelnik JF, Brown DM, Schmidt-Erfurth U, Do DV, Midena E, et al. Intravitreal aflibercept for diabetic macular edema: 148-week results from the VISTA and VIVID studies. Ophthalmology. 2016;123:2376-85.

14. Diabetic Retinopathy Clinical Research Network, Wells JA, Glassman AR, Ayala AR, Jampol LM, Aiello LP, Antoszyk AN, et al. Aflibercept, bevacizumab, or ranibizumab for diabetic macular edema. N Engl J Med. 2015;372:1193-203.

15. Korobelnik JF, Daien V, Faure C, Tadayoni R, Giocanti-Auregan A, Dot C, et al. Realworld outcomes following 12 months of intravitreal aflibercept monotherapy in patients with diabetic macular edema in France: results from the APOLLON study. Graefes Arch Clin Exp Ophthalmol. 2020;258:521-8.

16. Stefanickova J, Cunha-Vaz J, Ulbig M, Pearce I, Fernández-Vega Sanz A, Theodossiadis $P$, et al. A noninterventional study to monitor patients with diabetic macular oedema starting treatment with ranibizumab (POLARIS). Acta Ophthalmol. 2018;96:e942-9.

17. Ziemssen F, Schlottman PG, Lim Jl, Agostini H, Lang GE, Bandello F. Initiation of intravitreal aflibercept injection treatment in patients with diabetic macular edema: a review of VIVID-DME and VISTA-DME data. Int J Retina Vitreous. 2016;2:16.

18. Baker CW, Glassman AR, Beaulieu WT, Antoszyk AN, Browning DJ, Chalam KV, et al. Effect of initial management with aflibercept vs laser photocoagulation vs observation on vision loss among patients with diabetic macular edema involving the center of the macula and good visual acuity: a randomized clinical trial. JAMA. 2019;321:1880-94.

19. Ciulla TA, Pollack JS, Williams DF. Visual acuity outcomes and anti-VEGF therapy intensity in diabetic macular oedema: a real-world analysis of 28658 patient eyes. Br J Ophthalmol. 2021;105:216-21.

20. Bhandari S, Nguyen V, Fraser-Bell S, Mehta H, Viola F, Baudin F, et al. Ranibizumab or aflibercept for diabetic macular edema: comparison of 1-Year outcomes from the Fight Retinal Blindness! Registry. Ophthalmology. 2020;127:608-15. 
21. Lukic M, Williams G, Shalchi Z, Sim D, Patel PJ, Keane PA, et al. Intravitreal aflibercept for diabetic macular oedema: Moorfields' real-world 12-month visual acuity and anatomical outcomes. Eur J Ophthalmol. 2020;30:557-62.

\section{ACKNOWLEDGEMENTS}

The DRAKO study was sponsored by Bayer Plc. Medical writing support was provided by Andrea Parke of $\mathrm{O} 4$ Research and Apothecom Publications Ophthalmology, and was funded by Bayer Plc. The DRAKO Study Group on behalf of Bayer Plc. would like to thank the patients and investigators who have participated in the study.

\section{AUTHOR CONTRIBUTIONS}

SS, FG, SPK, AK, JT and PS were participating investigators in the study, played an important role in interpreting the results and form part of the steering committee. They reviewed, revised, provided feedback and approval of the manuscript, approve the decision to submit for publication and agree to be accountable for all aspects of the work. HM, AN, MS and JN participated in the study design, reviewed, revised, provided feedback and approval of the manuscript, approve the decision to submit the manuscript for publication and agree to be accountable for all aspects of the work.

\section{FUNDING}

This study was sponsored by Bayer Plc, Reading. The sponsor participated in the design of the study, conducting of the study, data collection, data management, data analysis, data interpretation and preparation, review and approval of the manuscript.

\section{COMPETING INTERESTS}

SS received consultancy fees from Bayer, Allergan, Novartis Pharma AG, Roche, Boehringer Ingelheim, Optos, Apellis, Oxurion, Biogen, Oculis and Heidelberg Engineering. SS is Editor-in-Chief for the Journal Eye. Faruque Ghanchi has been a consultant and speaker for Novartis, Bayer, Allergan, Alimera and Roche; and has received travel grants from Bayer and Novartis. SPK received travel grants from Bayer, research support from Bayer and Novartis Pharma AG, and participated in advisory boards for Novartis Pharma AG and Polyphotopics. AK received travel support from
Novartis, Bayer, and Allergan, and speaker fees from Allergan and Bayer. JT participated in advisory boards for Bayer and Novartis; received travel support from Bayer; and received research grants from Bayer, Novartis and Roche. PS attended advisory boards for Pfizer, Allergan, Boehringer, Roche and Bayer, and his department has received educational, research, and audit grants from Allergan, Boehringer Ingelheim, Novartis, and Bayer. HM is an employee of Bayer Plc. AN is an employee of Bayer Plc. MS is an employee of O4 Research. JN is an employee of Bayer Plc.

\section{ADDITIONAL INFORMATION}

Supplementary information The online version contains supplementary materia available at https://doi.org/10.1038/s41433-021-01624-9.

Correspondence and requests for materials should be addressed to S.S.

Reprints and permission information is available at http://www.nature.com/ reprints

Publisher's note Springer Nature remains neutral with regard to jurisdictional claims in published maps and institutional affiliations.

(i) Open Access This article is licensed under a Creative Commons Attribution 4.0 International License, which permits use, sharing, adaptation, distribution and reproduction in any medium or format, as long as you give appropriate credit to the original author(s) and the source, provide a link to the Creative Commons license, and indicate if changes were made. The images or other third party material in this article are included in the article's Creative Commons license, unless indicated otherwise in a credit line to the material. If material is not included in the article's Creative Commons license and your intended use is not permitted by statutory regulation or exceeds the permitted use, you will need to obtain permission directly from the copyright holder. To view a copy of this license, visit http://creativecommons. org/licenses/by/4.0/.

(c) The Author(s) 2021 